VOL. 53 (1996) [501-513]

\title{
OBLIQUE DERIVATIVE PROBLEM FOR QUASILINEAR ELLIPTIC EQUATIONS WITH VMO COEFFICIENTS
}

\author{
Guiseppe Di Fazio and Dian K. Palagachev
}

Strong solvability and uniqueness in the Sobolev space $W^{2, q}(\Omega), q>n$, are proved for the oblique derivative problem

$$
\begin{cases}\sum_{i, j=1}^{n} a^{i j}(x, u) D_{i j} u+b(x, u, D u)=0 & \text { almost everywhere in } \Omega \\ \partial u / \partial \ell+\sigma(x) u=\varphi & \text { on } \partial \Omega\end{cases}
$$

assuming the coefficients of the quasilinear elliptic operator to be Carathéodory functions, $a^{i j} \in V M O \cap L^{\infty}$ with respect to $x$, and $b$ to grow at most quadratically with respect to the gradient.

\section{INTRODUCTION}

In this paper we are concerned with strong solvability and uniqueness results for the regular oblique derivative problem for second order quasilinear elliptic equations. More precisely, we deal with the uniformly elliptic operator

$$
\mathcal{Q} u \equiv \sum_{i, j=1}^{n} a^{i j}(x, u) \frac{\partial^{2} u}{\partial x_{i} \partial x_{j}}+b(x, u, D u)
$$

in a bounded domain $\Omega \subset \mathbf{R}^{n}$, and first order boundary operator of the form

$$
\mathcal{B} u \equiv \sum_{i=1}^{n} \ell^{i}(x) \frac{\partial u}{\partial x_{i}}+\sigma(x) u,
$$

supposing the unit vector field $\ell(x)=\left(\ell^{1}(x), \ldots, \ell^{n}(x)\right)$ that prescribes $\mathcal{B}$ to be Lipschitz continuous and nowhere tangential to the boundary $\partial \Omega$. The coefficients $a^{i j}(x, z)$ and $b(x, z, p)\left((x, z, p) \in \Omega \times \mathbf{R} \times \mathbf{R}^{n}\right)$ of $\mathcal{Q}$ are assumed to satisfy Carathéodory's condition.

Received 18th August, 1995

The results presented here were obtained while the second author was visiting the Department of Mathematics of Catania University. The authors wish to express their deep gratitude to Filippo Chiarenga for the encouragement and the helpful discussions we had with him.

Copyright Clearance Centre, Inc. Serial-fee code: 0004-9729/96 \$A2.00+0.00. 
Our main goal here is to prove existence and uniqueness of a strong solution to the oblique derivative problem

$$
\mathcal{Q} u=0 \quad \text { almost everywhere in } \Omega, \quad \mathcal{B} u=\varphi(x) \quad \text { on } \partial \Omega
$$

As usual, by a strong solution of (1.1) we mean a function $u(x)$ belonging to the Sobolev space $W^{2, q}(\Omega)$ with a suitable $q \geqslant 1$ that satisfies the equation $\mathcal{Q} u=0$ almost everywhere in $\Omega$ and such that the boundary condition $\mathcal{B} u=\varphi$ holds in the trace sense on $\partial \Omega$.

The classical oblique derivative problem (1.1) with Hölder continuous coefficients has been extensively studied (see [6] and [10] for quasilinear $\mathcal{Q}$, and [11] for the general fully nonlinear case). Further, strong solvability for (1.1) was proved by Amann and Crandall [2] in the case of a semilinear operator $\mathcal{Q}\left(a^{i j}(x, z)=a^{i j}(x)\right)$ with uniformly continuous principal coefficients $a^{i j}(x)$. All these results assume the nonlinear term $b(x, z, p)$ to grow at most quadratically with respect to the gradient. On the other hand, a classical result due to Miranda [14] asserts $W^{2,2}$ strong solvability for the problem (1.1) if $a^{i j}(x) \in W^{1, n}$ and strictly sub-quadratic growth in $b(x, z, p)$ with respect to $p$ is assumed.

The aim of the present paper is to investigate the problem (1.1) weakening the regularity assumptions on the principal coefficients $a^{i j}(x, z)$ with respect to $x$. More precisely, we suppose these coefficients to belong to the class of functions with vanishing mean oscillation ( $V M O$ ) locally uniformly in $z$ (see $[16,3,4]$ ). The natural background for these considerations is the $L^{p}$ theory of the oblique derivative problem for linear elliptic equations with $V M O \cap L^{\infty}$ coefficients developed in [5]. We assume the nonlinear term $b(x, z, p)$ grows quadratically with respect to the gradient. It is worth noting that both the cases $a^{i j} \in C^{0}(\Omega)$ and $a^{i j} \in W^{1, n}(\Omega)$ imply $a^{i j} \in V M O$ (see [3]). In such a way, our result generalises those proved by Miranda [14] and Amann and Crandall [2].

The main tool in proving existence for the problem (1.1) is Leray and Schauder's fixed point theorem that reduces the solvability of (1.1) to the establishment of an a priori estimate in the space $W^{1,2 q}(\Omega), q>n$, for all possible solutions of a family of problems related to (1.1). We split the proof of that estimate into three steps. The first one is devoted to a bound for $\|u\|_{L^{\infty}(\Omega)}$ and this follows easily from a variant of Aleksandrov-Bakelman-Pucci maximum principle proved by Trudinger [17] (see [12, 13]). To apply the $L^{p}$-theory [5] of linear elliptic operators with $V M O$ coefficients, we must control the VMO-modulus of the composition $a^{i j}(x, u(x))$. Due to a simple result on composition, that control is equivalent to an a priori bound for suitable Hölder norm of $u$ which follows from Harnack type inequalities (see [11]). Finally, in order to estimate the $L^{2 q}(\Omega)$ norm of the gradient $D u$ we employ an approach inspired by 
Amann and Crandall [2]. The idea is to imbed our problem (1.1) into a family of similar problems depending on a parameter $\rho \in[0,1]$ and having solutions $u(\rho ; x)$. Then the norm $\|D u\|_{L^{2 q}(\Omega)}=\left\|D_{x} u(1 ; x)\right\|_{L^{2 q}(\Omega)}$ can be estimated in terms of $\left\|D_{x} u(0 ; x)\right\|_{L^{2 q}(\Omega)}$ after iterations on $\rho$, assuming the difference $u\left(\rho_{1} ; x\right)-u\left(\rho_{2} ; x\right)$ to be under control for small $\rho_{1}-\rho_{2}$. The crucial step in these investigations is ensured by the $L^{p}$ theory [5] of the oblique derivative problem for linear elliptic equations with $V M O \cap L^{\infty}$ coefficients.

At the end, the uniqueness result is a consequence of the Aleksandrov, Bakelman and Pucci maximum principle (Theorem 2.6 .2 in [17]), assuming in addition $b(x, z, p)$ to be Lipschitz continuous with respect to $z$ and $p$.

\section{Statement of the PRoblem and main ResUlts}

Let $\Omega \subset \mathbf{R}^{n}, n \geqslant 3$, be a bounded domain. The symbol $W^{k, q}(\Omega), k$ an integer, stands for the usual Sobolev space of $k$-times weakly differentiable functions with $L^{q}$-summable derivatives $D^{\alpha} u(|\alpha| \leqslant k)$, while $W^{s, q}(\partial \Omega), s>0$ a non integer, denotes the Besov space of functions defined on the boundary $\partial \Omega$ of $\Omega$ (see [1]). We adopt here the standard summation convention and $D_{i}=\partial / \partial x_{i}$, $D_{i j}=\partial^{2} / \partial x_{i} \partial x_{j}, D u=\left(D_{1} u, \ldots, D_{n} u\right)$. On the boundary $\partial \Omega$ is defined a unit vector field $\ell(x)=\left(\ell^{1}(x), \ldots, \ell^{n}(x)\right)$ that is nowhere tangential to $\partial \Omega$.

Our aim in the present paper is to study strong solvability of the oblique derivative problem

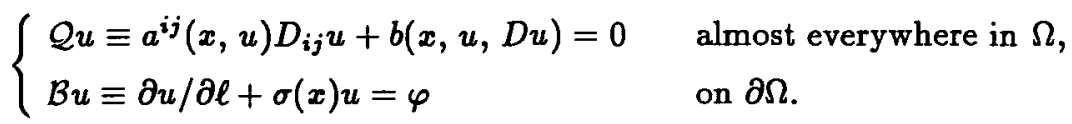

We shall treat here a uniformly elliptic operator $\mathcal{Q}$ and oblique operator $\mathcal{B}$ that satisfy certain regularity and structural conditions. Throughout the paper we assume the functions $a^{i j}(x, z)$ and $b(x, z, p)$ to be Carathéodory functions, that is, they are measurable with respect to $x \in \Omega$ for all $(z, p) \in \mathbf{R} \times \mathbf{R}^{n}$ and continuous in $z$ and $p$ for almost all $x \in \Omega$. Letting $\lambda$ denote a positive and non-increasing function, and $\eta_{K}, \omega_{K}$ and $\mu$ positive and non-decreasing functions we may list our assumptions as follows:

$a^{i j}(x, z) \xi^{i} \xi^{j} \geqslant \lambda(|z|)|\xi|^{2} \quad$ for almost all $x \in \Omega, \forall z \in \mathbf{R}, \forall \xi \in \mathbf{R}^{n}$ and $a^{i j}=a^{j i}$

$a^{i j}(x, z)$ is a VMO function with respect to $x$, locally uniformly in $z$ :

$$
\sup _{\rho \leqslant r} \frac{1}{\left|\Omega_{\rho}\right|} \int_{\Omega_{\rho}}\left|a^{i j}(x, z)-\frac{1}{\left|\Omega_{\rho}\right|} \int_{\Omega_{\rho}} a^{i j}(y, z) d y\right| d x=\eta_{K}(r) \longrightarrow 0 \quad \text { as } r \rightarrow 0
$$


for all $z \in[-K, K]$, where $\Omega_{\rho}=\Omega \cap B_{\rho}$ and $B_{\rho}$ ranges over the class of the balls with radius $\rho$ and centred at the points of $\Omega$ (we refer to $\eta_{K}(r)$ as the $V M O$-modulus of $\left.a^{i j}\right)$;

the coefficients $a^{i j}(x, z)$ are locally uniformly continuous with respect to $z$, uniformly in $\boldsymbol{x}$ :

$\left|a^{i j}(x, z)-a^{i j}\left(x, z^{\prime}\right)\right| \leqslant a(x) \omega_{K}\left(\left|z-z^{\prime}\right|\right) \quad$ almost everywhere in $\Omega, \forall z, z^{\prime} \in[-K, K]$ with $a(x) \in L^{\infty}(\Omega), \lim _{t \rightarrow 0} \omega_{K}(t)=0$ and $a^{i j}(x, 0) \in L^{\infty}(\Omega)$;

the function $b(x, z, p)$ grows at most quadratically with respect to the gradient:

$$
|b(x, z, p)| \leqslant \mu(|z|)\left(c(x)+|p|^{2}\right)
$$

for almost all $x \in \Omega, \forall(z, p) \in \mathbf{R} \times \mathbf{R}^{n}, c(x) \in L^{q}(\Omega), q>n$;

$b(x, z, p)$ is "monotone" with respect to $z$ :

$$
\operatorname{sign} z . b(x, z, p) \leqslant n\left\{\frac{g(x) \operatorname{det}\left[a^{i j}(x, z)\right]}{h(p)}\right\}^{1 / n}
$$

for almost all $x \in \Omega,|z| \geqslant M=$ const $>0, \forall p \in \mathbf{R}^{n}$, where $g \in L^{1}(\Omega), h \in L_{\text {loc }}^{1}\left(\mathbf{R}^{n}\right)$ are positive functions such that $\int_{\Omega} g(x) d x<\int_{\mathbb{R}^{n}} h(p) d p$.

The boundary operator $\mathcal{B}$ prescribed in terms of the directional derivative with respect to the unit vector field $\ell(x)=\left(\ell^{1}(x), \ldots, \ell^{n}(x)\right)$ we assume to be a regular boundary operator:

$$
\left\{\begin{array}{lc}
\ell(x) \cdot \nu(x)=\ell^{i}(x) \nu^{i}(x) \geqslant \kappa=\text { const }>0 & \text { on } \partial \Omega, \quad \sigma(x) \leqslant-\sigma_{0}=\text { const }<0, \\
\ell^{i}(x), \sigma(x) \in C^{0,1}(\partial \Omega), & \partial \Omega \in C^{1,1}
\end{array}\right.
$$

where $\nu(x)=\left(\nu^{1}(x), \ldots, \nu^{n}(x)\right)$ is the unit inner normal to $\partial \Omega$ and $C^{0,1}$ denotes the space of Lipschitz continuous functions.

We are now in a position to state our existence result.

THEOREM 2.1. Let conditions (2.2) - (2.7) be satisfied. Then for each $\varphi \in$ $W^{1-1 / q, q}(\partial \Omega)$, where $q>n$ is the number in (2.5), the problem (2.1) admits a strong solution $u \in W^{2, q}(\Omega)$. In particular, $u \in C^{1,1-n / q}(\bar{\Omega})$.

Proof: As usual, Theorem 2.1 will be proved with the aid of the Leray and Schauder fixed point theorem (see [8, Theorem 11.3]). With this goal, we define a nonlinear operator $\mathcal{F}$ whose fixed points will be solutions of the problem (2.1) as follows. Let $v \in W^{1,2 q}(\Omega)$. Then, $v \in C^{0}(\Omega)(q>n)$ and $a^{i j}(x, v(x)) \in V M O \cap L^{\infty}(\Omega)$ as 
a consequence of Proposition 3.3 below. Moreover, (2.5) leads to $b(x, v(x), D v(x)) \in$ $L^{q}(\Omega)$. Applying now the solvability result for the oblique derivative problem for linear elliptic equations with $V M O$ principal coefficients [5, Theorem 1.2] we conclude the existence of a unique strong solution $u(x) \in W^{2, q}(\Omega)$ of the linear problem:

$$
\begin{cases}a^{i j}(x, v(x)) D_{i j} u=-b(x, v(x), D v(x)) & \text { almost everywhere } \Omega \\ \partial u / \partial \ell+\sigma(x) u=\varphi & \text { on } \partial \Omega\end{cases}
$$

The nonlinear mapping

$$
\mathcal{F}: W^{1,2 q}(\Omega) \longrightarrow W^{2, q}(\Omega) \hookrightarrow W^{1,2 q}(\Omega)
$$

is defined by the formula $\mathcal{F} v=u$. Indeed, by virtue of Sobolev's imbedding theorem, $\mathcal{F}$ is a compact operator considered as a mapping from $W^{1, \dot{q} q}(\Omega)$ into itself. To prove the continuity of $\mathcal{F}$ let us set $u_{k}=\mathcal{F}_{k}, u=\mathcal{F} v$, where $v_{k} \rightarrow v$ in $W^{1,2 q}(\Omega)$ as $k \rightarrow \infty$. The difference $u_{k}-u \in W^{2, q}(\Omega)$ satisfies the equation

$$
a^{i j}(x, v) D_{i j}\left(u_{k}-u\right)=b(x, v, D v)-b\left(x, v_{k}, D v_{k}\right)+D_{i j} u_{k}\left(a^{i j}(x, v)-a^{i j}\left(x, v_{k}\right)\right)
$$

almost everywhere in $\Omega$ and satisfies the boundary condition

$$
\frac{\partial\left(u_{k}-u\right)}{\partial \ell}+\sigma(x)\left(u_{k}-u\right)=0 \quad \text { on } \partial \Omega
$$

It follows from Proposition 3.3 below, the linear a priori estimate [5, Theorem 1.2] and the maximum principle for the oblique derivative problem $([17$, Theorem 2.6.2], [13, Theorem 3.1], or [12, Theorem 1.5]) that

$$
\begin{aligned}
& \left\|u_{k}-u\right\|_{W^{2}, q(\Omega)} \\
\leqslant & C\left(\left\|b\left(x, v_{k}, D v_{k}\right)-b(x, v, D v)\right\|_{L^{q}(\Omega)}+\left\|D_{i j} u_{k}\left(a^{i j}\left(x, v_{k}\right)-a^{i j}(x, v)\right)\right\|_{L^{q}(\Omega)}\right) .
\end{aligned}
$$

The first term in the right-hand side above tends to 0 as $k \rightarrow \infty$ by means of (2.5) and the fact that the Nemytskii operator defined by $b$ is a continuous mapping [7, Theorem 16.11]. The same conclusion holds true on the second term because of the boundedness of $\left\|D_{i j} u_{k}\right\|_{L^{q}(\Omega)},(2.4)$ and $\left\|v_{k}-v\right\|_{L^{\infty}(\Omega)} \rightarrow 0(q>n)$. Therefore $u_{k} \rightarrow u$ in $W^{2, q}(\Omega)$ as $k \rightarrow \infty$. That proves the continuity of $\mathcal{F}$ by virtue of the continuity of the imbedding $W^{2, q}(\Omega) \hookrightarrow W^{1,2 q}(\Omega)$. Finally, in order to apply the Leray-Schauder fixed point theorem, that is, to assert existence of solution of the problem (2.1), it remains to prove the a priori estimate

$$
\|u\|_{W^{1,2 q(\Omega)}} \leqslant C
$$


with a constant $C$ independent of $u \in W^{1,2 q}(\Omega)$ and $\tau \in[0,1]$, for each solution $u$ of the operator equation $u=\tau \mathcal{F} u$, that is equivalent to the problem

$$
\begin{cases}a^{i j}(x, u) D_{i j} u+\tau b(x, u, D u)=0 & \text { almost everywhere in } \Omega \\ \partial u / \partial \ell+\sigma(x) u=\tau \varphi & \text { on } \partial \Omega\end{cases}
$$

The desired a priori estimate, however, is a consequence of Lemmas 3.1, 3.2 and 3.4 proved in Section 3 and thus the existence of a strong solution $u \in W^{2, q}(\Omega)$ to the problem (2.1) follows from the Leray-Schauder theorem. Finally, $u \in C^{1,1-n / q}(\bar{\Omega})$ as consequence of Morrey's lemma. This completes the proof of Theorem 2.1.

Uniqueness of the solution to the oblique derivative problem (2.1) can be proved in the wider class $W_{\text {loc }}^{2, n}(\Omega) \cap C^{1}(\Omega)$ in a special case concerning the structure of the coefficients and the nonlinear term of $\mathcal{Q}$.

THEOREM 2.2. Let the principal coefficients $a^{i j}$ of the operator $\mathcal{Q}$ be bounded and measurable functions that are independent of $z$, and assume conditions (2.2) (with $\lambda=$ const $>0)$ and (2.7) to be satisfied. Suppose further that $b(x, z, p)$ is nonincreasing function with respect to $z$ for almost all $x \in \Omega, \forall p \in \mathbf{R}^{n}$, and let $b(x, z, p)$ be Lipschitz continuous with respect to $z$ and $p$ :

$$
\left\{\begin{aligned}
&\left|b(x, z, p)-b\left(x, z^{\prime}, p\right)\right| \leqslant \beta_{1}\left(x, z, z^{\prime}, p\right)\left|z-z^{\prime}\right| \\
& \text { for almost all } x \in \Omega, \forall z, z^{\prime} \in \mathbf{R}, \forall p \in \mathbf{R}^{n}, \\
&\left|b(x, z, p)-b\left(x, z, p^{\prime}\right)\right| \leqslant \beta_{2}\left(x, z, p, p^{\prime}\right)\left|p-p^{\prime}\right| \\
& \text { for almost all } x \in \Omega, \forall z \in \mathbf{R}, \forall p, p^{\prime} \in \mathbf{R}^{n},
\end{aligned}\right.
$$

where $\sup _{|z|+\left|z^{\prime}\right|+|p| \leqslant K} \beta_{1}\left(\cdot, z, z^{\prime}, p\right) \in L^{n}(\Omega), \sup _{|z|+|p|+\left|p^{\prime}\right| \leqslant K} \beta_{2}\left(\cdot, z, p, p^{\prime}\right) \in L^{n}(\Omega)$, $K>0$.

Then, if $u_{1}, u_{2} \in W_{\text {loc }}^{2, n}(\Omega) \cap C^{1}(\Omega)$ are two solutions of the oblique derivative problem (2.1), we have $u_{1} \equiv u_{2}$ on $\bar{\Omega}$.

Proof: The difference $u=u_{1}-u_{2} \in W_{\mathrm{loc}}^{2, n}(\Omega) \cap C^{1}(\bar{\Omega})$ solves the linear oblique derivative problem

$$
\begin{cases}a^{i j}(x) D_{i j} u+B^{i}(x) D_{i} u+C(x) u=0 & \text { almost everywhere } \Omega \\ \partial u / \partial \ell+\sigma(x) u=0 & \text { on } \partial \Omega\end{cases}
$$

where

$$
\begin{aligned}
B^{i}(x) & =\int_{0}^{1} \frac{\partial b}{\partial p_{i}}\left(x, u_{1}(x), s D u(x)+D u_{2}(x)\right) d s \\
C(x) & =\int_{0}^{1} \frac{\partial b}{\partial z}\left(x, s u(x)+u_{2}(x), D u_{2}(x)\right) d s
\end{aligned}
$$


Indeed, the partial derivatives $\partial b / \partial p_{i}$ and $\partial b / \partial z$ exist almost everywhere in $\Omega \times \mathbf{R} \times \mathbf{R}^{n}$ by virtue of the Rademacher theorem and (2.10). Moreover, $B^{i}, C \in L^{n}(\Omega)$ and $C(x) \leqslant$ 0 since $b(x, z, p)$ is a non-increasing function with respect to $z$. $\bar{\Omega}$.

Therefore, (2.7) and the maximum principle [17, Theorem 2.6.2] imply $u \equiv 0$ on

\section{A PRIORI ESTIMATES}

This section is devoted to deriving the a priori estimate (2.8) with a constant $C$ independent of $u$ and $\tau$, for all possible solutions of the problem (2.9). This procedure is a three-step process involving successive estimation of $\|u\|_{L^{\infty}(\Omega)}$, the modulus of continuity of $u$ and $\|D u\|_{L^{2 q(\Omega)}}$, and each of these estimates presupposes the preceding ones. Let us note that the bound on $\|u\|_{L^{\infty}(\Omega)}$ is necessary in order to control behaviour with respect to $z$ in $a^{i j}(x, z)$ and $b(x, z, p)$. Further, the information on the modulus of continuity of $u$ allows us to control the $V M O$-modulus of the composition $a^{i j}(x, u(x))$ (the function $\eta_{K}(r)$ with $K=\|u\|_{L^{\infty}(\Omega)}$, in (2.3)) through a simple result on composition (Proposition 3.3).

A maximum principle of Aleksandrov-Bakelman-Pucci type is the main tool in estimating $\|u\|_{L^{\infty}(\Omega)}$. To control the modulus of continuity of $u(x)$ on $\bar{\Omega}$, we shall derive stronger estimate on the norm of $u$ in a Holder space $C^{0, \gamma}(\bar{\Omega})$ with a suitable $\gamma \in(0,1)$. This bound for the Hölder norm (see [11]) is obtained by a technique due to Krylov and Safonov which generalises classical estimates of De Giorgi-NashMoser, and it is based on the weak Harnack inequalities. Finally, the a priori bound for $\|D u\|_{L^{2 q}(\Omega)}$ is derived after embedding our problem into a one-parameter family of related oblique derivative problems and iterating with respect to the parameter the $L^{2 q_{-}}$ norms of the gradients. This method is inspired by Amann and Crandall's approach (see [2]) in obtaining $L^{\infty}$-gradient estimates for semilinear elliptic equations with continuous coefficients.

Before starting to realise our program, let us note that we must prove estimate (2.8) for each possible solution of problem (2.1) instead of (2.9) since $\tau \in[0,1]$. The letter $C$ will denote a constant, independent of $u$, that may vary from line to line.

\subsection{A PRIORI ESTIMATE FOR $\|u\|_{L^{\infty}(\Omega)}$.}

LEMMA 3.1. Suppose conditions (2.2), (2.6) and (2.7) are satisfied. Then

$$
\|u\|_{L^{\infty}(\Omega)} \leqslant \max \left\{M, \frac{\max _{\theta \Omega}|\varphi(x)|}{\sigma_{0}}\right\}+R\left\{\frac{1}{\sigma_{0}}+\operatorname{diam} \Omega\right\}
$$

for each solution $u \in W^{2, q}(\Omega)$ of the problem (2.1), where $R$ is given by

$$
\int_{B_{R}(0)} h(p) d p=\int_{\Omega} g(x) d x \quad \text { and } \quad B_{R}(0)=\left\{p \in \mathbf{R}^{n}:|p|<R\right\} .
$$


Proof: This is a direct consequence of Theorem 2.6.1 in Trudinger's Lecture Notes [17]. The only remark we have to point out is that $\varphi \in W^{1-1 / q, q}(\partial \Omega)$ and $q>n$ imply $\varphi \in C^{0}(\partial \Omega)$.

\subsection{A PRIORI ESTIMATE FOR THE MODULUS OF CONTINUITY OF $u$.}

LEMMA 3.2. Let $u \in W^{2, q}(\Omega)$ be a strong solution of the oblique derivative problem (2.1) and suppose conditions (2.2), (2.4), (2.5) and (2.7) are satisfied. Then

$$
\sup _{x \neq y, x, y \in \bar{\Omega}} \frac{|u(x)-u(y)|}{|x-y|^{\gamma}} \leqslant C
$$

where $\gamma \in(0,1)$ is a constant depending on $n, q, \lambda\left(\|u\|_{L^{\infty}(\Omega)}\right), \mu\left(\|u\|_{L^{\infty}(\Omega)}\right), \kappa$, $\left\|a^{i j}(x, 0)\right\|_{L^{\infty}(\Omega)}$ and $C$ depends in addition on $\|c\|_{L^{q}(\Omega)}, \sigma(x), \varphi(x), \partial \Omega$.

ProOF: The estimate (3.1) follows in the same manner as in the proof of [17, Theorem 2.3] using interior and boundary inequalities of Harnack type. Due to the fact that we are dealing with strong solutions, however, a maximum principle of AleksandrovBakelman-Pucci type ([12, Theorem1.5], [17, Theorem 2.6.1]) is to be used instead of the classical maximum principle.

3.4 A PRIORI ESTIMATE FOR $\|D u\|_{L^{2 q}(\Omega)}$.

As mentioned above, the desired bound for $\|D u\|_{L^{2 q}(\Omega)}$ will be derived by employing an approach inspired by the method of Amann and Crandall (see [2]) in proving an $L^{\infty}(\Omega)$-gradient estimate for semilinear elliptic equations. However, in order to be able to apply the linear theory developed in [5], first of all we must prove the following simple result on composition.

Proposition 3.3. Let assumptions (2.3) and (2.4) be satisfied and suppose $u(x) \in C^{0}(\Omega)$. Then the composition $a^{i j}(x, u(x)) \in V M O \cap L^{\infty}(\Omega)$ and its VMOmodulus is bounded in terms of $\|u\|_{L^{\infty}(\Omega)}$ and the continuity modulus of $u(x)$.

Proof: It follows from (2.4) that the composition $a^{i j}(x, u(x)) \in L^{\infty}(\Omega) \subset B M O$ (see [9]). Let $B_{\rho}$ be an arbitrary ball centred at a point $x_{0} \in \Omega$ and $\rho \leqslant r$. Then

$$
J(\rho)=\frac{1}{\left|\Omega_{\rho}\right|} \int_{\Omega_{\rho}}\left|a^{i j}(x, u(x))-\frac{1}{\left|\Omega_{\rho}\right|} \int_{\Omega_{\rho}} a^{i j}(y, u(y)) d y\right| d x \leqslant J_{1}(\rho)+J_{2}(\rho),
$$

with

$$
\begin{aligned}
& J_{1}(\rho)=\frac{2}{\left|\Omega_{\rho}\right|} \int_{\Omega_{\rho}}\left|a^{i j}(x, u(x))-a^{i j}\left(x, u\left(x_{0}\right)\right)\right| d x \\
& J_{2}(\rho)=\frac{1}{\left|\Omega_{\rho}\right|} \int_{\Omega_{\rho}}\left|a^{i j}\left(x, u\left(x_{0}\right)\right)-\frac{1}{\left|\Omega_{\rho}\right|} \int_{\Omega_{\rho}} a^{i j}\left(y, u\left(x_{0}\right)\right) d y\right| d x
\end{aligned}
$$


It follows from (2.4) that

$$
J_{1}(\rho) \leqslant 2\|a\|_{L^{\infty}(\Omega)} \omega_{\|u\|_{L^{\infty}(\Omega)}}(\theta(u, r))
$$

where $\theta(u, r)$ is the modulus of continuity of $u$, while (2.3) ensures

$$
J_{2}(\rho) \leqslant \eta_{\|u\|_{L} \infty(n)}(r)
$$

Therefore

$$
\sup _{\rho \leqslant r} J(\rho) \leqslant 2\|a\|_{L^{\infty}(\Omega)} \omega_{\|u\|_{L} \infty(\Omega)}(\theta(u, r))+\eta_{\|u\|_{L^{\infty}(\Omega)}}(r) \longrightarrow 0 \quad \text { as } r \rightarrow 0 .
$$

This proves $a^{i j}(x, u(x)) \in V M O$. Moreover the $V M O$-modulus of the composition is bounded in terms of $\|u\|_{L^{\infty}(\Omega)}$ and of a monotone function of the $u$-continuity modulus.

Lemma 3.4. Let conditions (2.2), (2.3), (2.4), (2.5) and (2.7) be satisfied. Then there exists a constant $C$ depending on known quantities only and on $\|u\|_{L^{\infty}(\Omega)}$, such that

$$
\|D u\|_{L^{2 q(\Omega)}} \leqslant C
$$

for each solution $u \in W^{2, q}(\Omega)$ of the oblique derivative problem (2.1).

Proof: The function $u \in W^{2, q}(\Omega)$ solves the equation

$$
A^{i j}(x) D_{i j} u+B(x)|D u|^{2}-c(x) u(x)=f(x) \quad \text { almost everywhere in } \Omega
$$

where

$$
\left\{\begin{aligned}
A^{i j}(x) & =a^{i j}(x, u(x)) \in V M O \cap L^{\infty}(\Omega) \quad \text { (by Proposition 3.3), } \\
B(x) & =\frac{b(x, u(x), D u(x))}{c(x)+|D u(x)|^{2}} \in L^{\infty}(\Omega), \\
f(x) & =-c(x) u(x)-\frac{c(x) b(x, u(x), D u(x))}{c(x)+|D u(x)|^{2}} \in L^{q}(\Omega) .
\end{aligned}\right.
$$

For the fixed solution $u(x)$ of the problem (2.1), we imbed (2.1) into the oneparameter family of oblique derivative problems

$\begin{cases}A^{i j}(x) D_{i j} u(\rho ; x)+B(x)|D u(\rho ; x)|^{2}-c(x) u(\rho ; x)=\rho f(x) & \text { almost everywhere in } \Omega, \\ \partial u(\rho ; x) / \partial \ell+\sigma(x) u(\rho ; x)=\rho \varphi & \text { on } \partial \Omega\end{cases}$ 
with solutions $u(\rho ; x) \in W^{2, q}(\Omega)(\rho \in[0,1])$ if they exist. Let us point out that $q$. $>n$ and Sobolev's imbedding theorem ensure that the boundary condition in (3.4) is satisfied in the classical sense on $\partial \Omega$.

Indeed, what we know about the problem (3.4) at this moment is that $u(0 ; x)=$ 0 and $u(1 ; x) \equiv u(x)$ is the fixed solution of (2.1). Our aim will be to estimate $\left\|D_{x} u\left(\rho_{2} ; x\right)\right\|_{L^{2 q}(\Omega)}$ in terms of $\left\|D_{x} u\left(\rho_{1} ; x\right)\right\|_{L^{2 q(\Omega)}}$ when $\rho_{2}-\rho_{1}>0$ is small enough. After that, assuming we have in addition unique solvability in $W^{2, q}(\Omega)$ of $(3.4)$ for each value $\rho \in[0,1]$, it will be easy to derive the desired estimate (3.2) by iteration of $L^{2 q}(\Omega)$-norms of $D_{x} u(\rho ; x)$ for $\rho<1$.

STEP 1. To realise our program, we shall estimate at first the difference of two solutions of the problem (3.4) in terms of the difference between the corresponding values of the parameter $\rho$. Let $u\left(\rho_{1} ; x\right), u\left(\rho_{2} ; x\right) \in W^{2, q}(\Omega)$ solve (3.4) with $\rho_{1} \leqslant \rho_{2}$. Then

$\left\|u\left(\rho_{1} ; x\right)-u\left(\rho_{2} ; x\right)\right\|_{L^{\infty}(\Omega)} \leqslant\left(\rho_{2}-\rho_{1}\right)\left(\|u\|_{L^{\infty}(\Omega)}+\mu\left(\|u\|_{L^{\infty}(\Omega)}\right)+\frac{1}{\sigma_{0}} \max _{\partial \Omega}|\varphi(x)|\right)$.

In order to prove (3.5), we set $w(x)=u\left(\rho_{1} ; x\right)-u\left(\rho_{2} ; x\right)$ and observe that $w \in$ $W^{2, q}(\Omega)$ solves the linearised oblique derivative problem

$\begin{cases}\mathcal{L} w \equiv A^{i j}(x) D_{i j} w+B^{i}(x) D_{i} w-c(x) w=\left(\rho_{1}-\rho_{2}\right) f(x) & \text { almost everywhere in } \Omega \\ \mathcal{M} w \equiv \partial w / \partial \ell+\sigma(x) w=\left(\rho_{1}-\rho_{2}\right) \varphi & \text { on } \partial \Omega,\end{cases}$

where

$$
B^{i}(x)=2 B(x) \int_{0}^{1}\left(s D_{i} w+D_{i} u\left(\rho_{2} ; x\right)\right) d s \in L^{q}(\Omega)
$$

On the other hand,

$$
\begin{aligned}
\mathcal{L} w & =\left(\rho_{1}-\rho_{2}\right) f(x) \geqslant-c(x)\left(\rho_{2}-\rho_{1}\right)\left(\|u\|_{L^{\infty}(\Omega)}+\mu\left(\|u\|_{L^{\infty}(\Omega)}\right)+\frac{1}{\sigma_{0}} \max _{\theta \Omega}|\varphi(x)|\right) \\
& =\mathcal{L}\left(\left(\rho_{2}-\rho_{1}\right)\left(\|u\|_{L^{\infty}(\Omega)}+\mu\left(\|u\|_{L^{\infty}(\Omega)}\right)+\frac{1}{\sigma_{0}} \max _{\theta \Omega}|\varphi(x)|\right)\right)
\end{aligned}
$$

as consequence of (3.3) and (2.5). Further,

$$
\begin{aligned}
\mathcal{M} w & =\left(\rho_{1}-\rho_{2}\right) \varphi(x) \geqslant\left(\rho_{2}-\rho_{1}\right) \sigma(x) \frac{1}{\sigma_{0}} \max _{\theta \Omega}|\varphi(x)| \\
& \geqslant\left(\rho_{2}-\rho_{1}\right) \sigma(x)\left(\|u\|_{L^{\infty}(\Omega)}+\mu\left(\|u\|_{L^{\infty}(\Omega)}\right)+\frac{1}{\sigma_{0}} \max _{\theta \Omega}|\varphi(x)|\right) \\
& =\mathcal{M}\left(\left(\rho_{2}-\rho_{1}\right)\left(\|u\|_{L^{\infty}(\Omega)}+\mu\left(\|u\|_{L^{\infty}(\Omega)}\right)+\frac{1}{\sigma_{0}} \max _{\theta \Omega}|\varphi(x)|\right)\right)
\end{aligned}
$$


on $\partial \Omega$.

Therefore, it follows from the maximum principle $[17$, Theorem 2.6.2] that

$$
\sup _{\Omega} w \leqslant\left(\rho_{2}-\rho_{1}\right)\left(\|u\|_{L^{\infty}(\Omega)}+\mu\left(\|u\|_{L^{\infty}(\Omega)}\right)+\frac{1}{\sigma_{0}} \max _{\delta \Omega}|\varphi(x)|\right) .
$$

Similarly, replacing $w$ by $-w$ above we can estimate inf $w$ from below. This leads to the bound (3.5).

REMARK 3.5. By taking $\rho_{1}=\rho_{2}$ in (3.5), we obtain immediately a uniqueness result for solutions of the problem (3.4) for each value $\rho \in[0,1]$.

STEP 2. Let $\rho_{1}<\rho_{2}$ be two arbitrary numbers and suppose there exist solutions $u\left(\rho_{1} ; x\right)$ and $u\left(\rho_{2} ; x\right) \in W^{2, q}(\Omega)$ of (3.4). The difference $w(x)=u\left(\rho_{1} ; x\right)-u\left(\rho_{2} ; x\right) \in$ $W^{2, q}(\Omega)$ solves the problem

$$
\begin{cases}A^{i j}(x) D_{i j} w=\left(\rho_{1}-\rho_{2}\right) f(x)-B(x)\left(\left|D u\left(\rho_{1} ; x\right)\right|^{2}-\left|D u\left(\rho_{2} ; x\right)\right|^{2}\right)+c(x) w \\ \partial w / \partial \ell+\sigma w=\left(\rho_{1}-\rho_{2}\right) \varphi & \text { almost everywhere in } \Omega, \\ & \text { on } \partial \Omega\end{cases}
$$

and the a priori estimate for solutions of the oblique derivative problem for linear elliptic equations with $V M O$ coefficients [5, Theorem 1.2] asserts

$$
\begin{gathered}
\|w\|_{W^{2, q}(\Omega)} \leqslant C\left(\left\|\left(\rho_{1}-\rho_{2}\right) f(x)-B(x)\left(\left|D u\left(\rho_{1} ; x\right)\right|^{2}-\left|D u\left(\rho_{2} ; x\right)\right|^{2}\right)+c(x) w\right\|_{L^{q}(\Omega)}\right. \\
\left.+\left\|\left(\rho_{1}-\rho_{2}\right) \varphi(x)\right\|_{W^{1-1 / q, q(g \Omega)}}\right)
\end{gathered}
$$

where the constant $C$ depends on known quantities and on $u$ through $\|u\|_{L^{\infty}(\Omega)}$ only.

Therefore,

$$
\|w\|_{W^{2, q(\Omega)}} \leqslant C\left(1+\left\|D u\left(\rho_{1} ; x\right)\right\|_{L^{2 q}(\Omega)}^{2}+\|D w\|_{L^{2 q}(\Omega)}^{2}\right)
$$

as a consequence of (2.5), (3.3) and (3.5), where $C$ depends on $\|u\|_{L^{\infty}(\Omega)}$ but it is independent of $\left(\rho_{1}-\rho_{2}\right)$.

On the other hand,

$$
\begin{aligned}
\|D w\|_{L^{2 q}(\Omega)}^{2} & \leqslant C\|w\|_{L^{\infty}(\Omega)}\left\|D^{2} w\right\|_{L^{q}(\Omega)} \\
& \leqslant C\left(\rho_{2}-\rho_{1}\right)\left(\|u\|_{L^{\infty}(\Omega)}+\mu\left(\|u\|_{L^{\infty}(\Omega)}\right)+\frac{1}{\sigma_{0}} \max _{\partial \Omega}|\varphi(x)|\right)\left\|D^{2} w\right\|_{L^{q}(\Omega)}
\end{aligned}
$$

by virtue of the Gagliardo-Nirenberg interpolation inequality [15] and the estimate (3.5). 
Thus, making use of (3.6), we have

$$
\|D w\|_{L^{2 q}(\Omega)}^{2} \leqslant C_{0}\left(1+\left(\rho_{2}-\rho_{1}\right)\|D w\|_{L^{2 q}(\Omega)}^{2}+\left\|D u\left(\rho_{1}, \cdot\right)\right\|_{L^{2 q}(\Omega)}^{2}\right)
$$

with a new constant $C_{0}$ that is independent of $\rho_{1}-\rho_{2}$.

Now, supposing that $\rho_{2}-\rho_{1} \leqslant \varepsilon$ where $C_{0} \varepsilon<1 / 2$, the last inequality leads to

$$
\|D w\|_{L^{2 q}(\Omega)}^{2} \leqslant C\left(1+\left\|D u\left(\rho_{1}, \cdot\right)\right\|_{L^{2 q(\Omega)}}^{2}\right),
$$

whence

$$
\left\|D u\left(\rho_{2}, \cdot\right)\right\|_{L^{2 q}(\Omega)}^{2} \leqslant C_{1}+C_{2}\left\|D u\left(\rho_{1}, \cdot\right)\right\|_{L^{2 q}(\Omega)}^{2}
$$

whenever $\rho_{2}-\rho_{1} \leqslant \varepsilon$.

Now, taking $\rho_{1}=0$ and $\rho_{2}=\varepsilon$ above, and bearing in mind the uniqueness result (Remark 3.5), we have

$$
\|D u(\varepsilon, \cdot)\|_{L^{2 q}(\Omega)}^{2} \leqslant C_{1}
$$

whenever there exists the solution $u(\varepsilon ; x) \in W^{2, q}(\Omega)$ of the problem (3.4) with $\rho=\varepsilon$.

STEP 3. To complete the proof of Lemma 3.4 it remains to show that the problem (3.4) is solvable in $W^{2, q}(\Omega)$ as $\rho=\varepsilon$. We shall prove this with the aid of the Leray-Schauder fixed point theorem again. For this goal, we define the compact nonlinear operator

$$
\mathcal{P}: W^{1,2 q}(\Omega) \longrightarrow W^{2, q}(\Omega) \hookrightarrow W^{1,2 q}(\Omega)
$$

in the following way: for each $v \in W^{1,2 q}(\Omega)$ the image $\mathcal{P} v \in W^{2, q}(\Omega)$ is the unique solution of the linear oblique derivative problem:

$$
\begin{cases}A^{i j}(x) D_{i j}(\mathcal{P} v)=\varepsilon f(x)-B(x)|D v|^{2}+c(x) v & \text { almost everywhere in } \Omega, \\ \partial(\mathcal{P} v) / \partial \ell+\sigma \mathcal{P} v=\varepsilon \varphi & \text { on } \partial \Omega\end{cases}
$$

Of course, $A^{i j} \in V M O \cap L^{\infty}(\Omega), \varepsilon f(x)-B(x)|D v|^{2}+c(x) v \in L^{q}(\Omega)$ and therefore the above problem is uniquely solvable in the space $W^{2, q}(\Omega)$ by virtue of $[5$, Theorem 1.2]. Moreover, the already cited result ensures that $\mathcal{P}$ is a continuous operator from $W^{1,2 q}(\Omega)$ into itself. Finally, the estimate (3.8) (with $\tau \varepsilon$ instead of $\varepsilon$ ) holds true for each solution of the equation $v=\tau \mathcal{P} v, \tau \in[0,1]$, that is equivalent to the problem

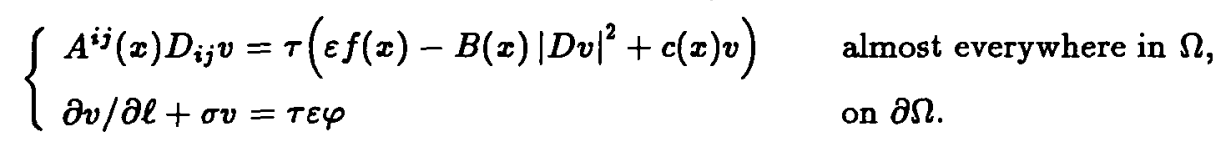

Thus the Leray-Schauder theorem asserts the existence of a fixed point of the mapping $\mathcal{P}$. This proves solvability in $W^{2, q}(\Omega)$ of $(3.4)$ in the case $\rho=\varepsilon$.

To complete the proof of Lemma 3.4 we put $\rho_{1}=k \varepsilon, \rho_{2}=(k+1) \varepsilon, k=1,2, \ldots$, in (3.7) and by applying finitely many times the above procedure, we get the desired estimate (3.2) for $u(x) \equiv u(1 ; x)$. 


\section{REFERENCES}

[1] R. Adams, Sobolev Spaces (Academic Press, New York, 1975).

[2] H. Amann and M. Crandall, 'On some existence theorems for semi-linear elliptic equations', Indiana Univ. Math. J. 27 (1978), 779-790.

[3] F. Chiarenza, M. Frasca and $P$. Longo, 'Interior $W^{2, p}$ estimates for non divergence elliptic equations with discontinuous coefficients', Ricerche Mat. 60 (1991), 149-168.

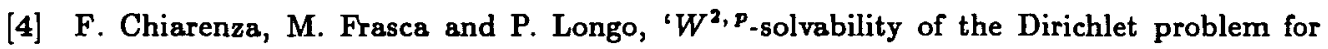
non divergence elliptic equations with VMO coefficients', Trans. Amer. Math. Soc. 336 (1993), 841-853.

[5] G. Di Fazio and D. K. Palagachev, 'Oblique derivative problem for elliptic equations in non-divergence form with VMO coefficients', Comment. Math. Univ. Caronlin. (to appear).

[6] R. Fiorenza,, 'Sui problemi di derivata obliqua per le equazioni ellittiche quasi lineari', Ricerche Mat. 15 (1966), 74-108.

[7] S. Fučik and A. Kufner, Nonlinear differential equations (Elsevier Sci. Publ. Co., New York, 1980).

[8] D. Gilbarg and N.S. Trudinger, Elliptic partial differential equations of second order, (2nd ed.) (Springer-Verlag, Berlin, Heidelberg, New York, 1983).

[9] F. John and L. Nirenberg, 'On functions of bounded mean oscillation', Comm. Pure Appl. Math. 14 (1961), 415-426.

[10] G.M. Lieberman, 'Solvability of quasilinear elliptic equations with nonlinear boundary conditions. II', J. Funct. Anal. 56 (1984), 210-219.

[11] G.M. Lieberman and N.S. Trudinger, 'Nonlinear oblique boundary value problem for nonlinear elliptic equations', Trans. Amer. Math. Soc. 295 (1986), 509-545.

[12] Y. Luo, 'An Aleksandrov-Bakelman type maximum principle and applications', J. Diff. Equations 101 (1993), 213-231.

[13] Y. Luo and N.S. Trudinger, 'Linear second order elliptic equations with Venttsel boundary conditions', Proc. Roy. Soc. Edinburgh Sect. A 118 (1991), 193-207.

[14] C. Miranda, 'Sulle equazioni ellittiche del secondo ordine di tipo non variazionale, a coefficienti discontinui', Ann. Mat. Pura Appl. 63 (1963), 353-386.

[15] L. Nirenberg, 'On elliptic partial differential equations', Ann. Scuola Norm. Sup. Pisa 13 (1959), 115-162.

[16] D. Sarason, 'Functions of vanishing mean oscillation', Trans. Amer. Math. Soc. 207 (1975), 391-405.

[17] N.S. Trudinger, Nonlinear second order elliptic equations, Lecture Notes of Math. Inst. of Nankai Univ. (Tianjin, China, 1986).

Department of Mathematics

University of Catania

Viale A. Doria 6

95125 Catania

Italy

e-mail-DiFaziodipmat.unict.it
Department of Mathematics

Technological University of Sofia

8 "Kl. Okhridski" blv

Sofia - 1756

Bulgaria

e-mail: dian@bgcict.acad.bg 